\title{
Linking the X-ray timing and spectral properties of the glitching AXP 1RXS J170849-400910
}

\author{
G. L. Israel ${ }^{1}$, D. Götz ${ }^{2}$, S. Zane ${ }^{3}$, S. Dall'Osso ${ }^{1}$, N. Rea ${ }^{4}$, and L. Stella ${ }^{1}$ \\ 1 INAF - Osservatorio Astronomico di Roma, via Frascati 33, 00040 Monte Porzio Catone, Italy \\ e-mail: gianluca@mporzio.astro.it \\ 2 CEA Saclay, DSM/DAPNIA/Service d'Astrophysique, 91191 Gif-sur-Yvette, France \\ 3 Mullard Space Science Laboratory University College of London Holmbury St Mary, Dorking, Surrey, RH5 6NT, UK \\ ${ }^{4}$ SRON - Netherlands Institute for Space Research, Sorbonnelaan 2, 3584 CA Utrecht, The Netherlands
}

Received 3 July 2007 / Accepted 24 October 2007

ABSTRACT

\begin{abstract}
Aims. Previous studies of the X-ray flux and spectral properties of 1RXS J170849-400910 showed a possible correlation with the spin glitches that occurred in 1999 and 2001. However, due to the sparseness of spectral measurements and the paucity of detected glitches, no firm conclusion could be drawn.

Methods. We retrieved and analysed archival Rossi-XTE pointings of 1RXS J170849-400910 covering the time interval between January 2003 and June 2006 and carried out a detailed timing analysis with phase fitting techniques.

Results. We detected two large glitches $\left(\frac{\Delta v}{v}\right.$ of 1.2 and $\left.2.1 \times 10^{-6}\right)$ that occurred in January and June 2005. The occurrence times of these glitches are in agreement with the predictions made in our previous studies. This finding strongly suggests a connection between the flux, spectral and timing properties of 1RXS J170849-400910.
\end{abstract}

Key words. stars: individual: 1RXS J170849-400910 - stars: neutron - stars: X-rays

\section{Introduction}

1RXS J170849-400910 (hereafter RXSJ1708) is one of the anomalous X-ray pulsars (AXPs), a small group of peculiar neutron stars (NSs) that are currently believed to have super-strong magnetic fields, $B \sim 10^{14}-10^{15} \mathrm{G}$, hence dubbed "magnetars" (though other possible models are not completely ruled out by observations; for a recent review see Woods \& Thompson 2006). RXSJ1708 was first discovered by ROSAT (Voges et al. 1996), while $\sim 11 \mathrm{~s}$ coherent pulsations were detected with ASCA (Sugizaki et al. 1997). Early measurements suggested that it was a fairly stable rotator, with a spin-down rate of $\sim 2 \times 10^{-11} \mathrm{~s} \mathrm{~s}^{-1}$, and a soft spectrum (Israel et al. 1999, 2001). Events of sudden spin-up (glitches) with very different post-glitch recovery were detected in RXSJ1708 by Rossi-XTE in 1999 and 2001 (Kaspi et al. 2000; Dall'Osso et al. 2003; Kaspi \& Gavriil 2003). The rather short interglitch time makes this AXP a frequent glitcher among neutron stars.

Recently, Rea et al. (2005a) noticed that the long term (over 5 years) variations in the source X-ray flux and spectral hardness are correlated, with both quantities reaching a maximum close to the epochs of the two glitches in 1999 and 2001. The addition of new data obtained from Chandra (2004) and Swift observations confirmed the flux-hardening correlation (Campana et al. 2007), extending its validity to hard X-rays (Götz et al. 2007). Following these studies, Zane et al. (2007) and Rea et al. (2007) suggested that the long-term variations may have a cyclic behavior with a recurrence time of $\approx 5-10$ yrs, possibly due to a periodic twisting/untwisting of the star magnetosphere (Beloborodov \& Thompson 2007). Correspondingly, the source was expected to re-enter into a glitching active phase during 2005-2006, close to the latest maximum in the source flux.
Thus, we analyzed the Rossi-XTE archival data spanning the latest 3.5 years and performed a phase coherent analysis of the pulse arrival times in a search for new glitches. We detected two large glitches occurring on January 2005 and May 2005, in agreement with the prediction of Zane et al. (2007) and Rea et al. (2007) and correlating with the flux and spectral evolution.

\section{Rossi-XTE observations and timing analysis}

We analyzed 204 Rossi-XTE archival observations of RXSJ1708 ${ }^{1}$. These span from 2003 January 5th to 2006 June 3rd. We restricted our analysis to the PCA instrument (Jahoda et al. 1996) which was operated in good Xenon data mode with a time resolution of $1 \mu$ s and 256 energy bins between 2 and $120 \mathrm{keV}$. Raw data were reduced using the ftools v6.2, provided by the High Energy Astrophysics Science Archive Research Center ${ }^{2}$. The events were extracted in the 2.5-16 keV energy range, and binned into light curves of $0.125 \mathrm{~s}$ resolution. Photon arrival time correction to the barycentric dynamical time (TDB) was applied by using fxbary and the $\left(\sim 1^{\prime \prime}\right.$ accurate) source position provided by Israel et al. (2003).

A phase-coherent timing solution was first obtained using a relatively long (29 ks) archival Chandra observations carried out on 2004 July 3rd (starting time at MJD 53 189.151489 TDB; for details on the data reduction of this dataset see Rea et al. $2005 b)$. This provided a sufficiently accurate period determination, $P=11.00231(3)$ s, such that no pulse cycle was missed

\footnotetext{
ftp://legacy.gsfc.nasa.gov/xte/data/archive

2 http://heasarc.gsfc.nasa.gov/docs/software/ftools/ ftools_menu.html
} 


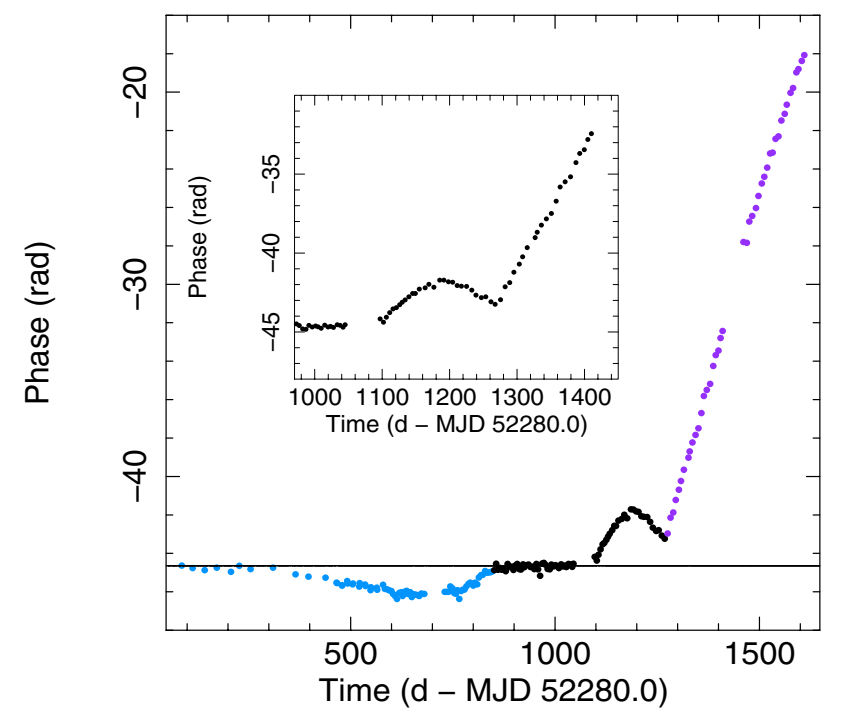

Fig. 1. Rossi-XTE time residuals for the time interval January 2003June 2006 after subtraction of the phase-coherent $P-\dot{P}$ solution inferred by Dall'Osso et al. (2003). The inset shows the time interval over which we detected the two glitches.

when extrapolating this value to the epoch of the closest RossiXTE pointing (2004 July 1st). A phase-coherent timing solution was inferred in the time interval between 2004 May 1st and November 16th corresponding to $v=0.090890035(1) \mathrm{Hz}$ and $\dot{v}=-1.5884(14)$ (epoch $53819.0 \mathrm{MJD} ; 1 \sigma$ c.l. are reported).

The inclusion of the 2005 and 2006 datasets showed large disagreement with the above inferred timing solution, with two evident "jumps" in phase both marking a decrease in the period value (see blue and dark violet filled circles in Fig. 1), strongly suggesting that two further glitches occurred at the end of 2004/begin 2005 (MJD 53370) and in May 2005 (MJD 53 550). No signature for similarly large glitches was instead found in the datasets spanning between January 2003 and June 2004. The phases reported in Fig. 1 are obtained using the Dall'Osso et al. (2003) 2001 post-glitch solution (horizontal solid line) which is nearly coincident to the phase coherent solution inferred above. Thus, we applied a detailed timing analysis to phase residuals were the two large glitches occurred. A more exhaustive study and modelling of the whole phase and glitch history of RXSJ1708 will be presented elsewhere (Dall'Osso et al. 2007). Following the analysis scheme outlined in Dall'Osso et al. (2003) we inferred the main parameters of the two detected large glitches (see Table 1). Figure 2 shows the phase residuals after subtraction of the timing solutions pre- and post-glitch N.1, and post-glitch N.2. Both the newly detected glitches reveal large jumps in the spindown rate, $\Delta \dot{v} / \dot{v} \sim 7 \times 10^{-2}$ and $\Delta \dot{v} / \dot{v} \sim-0.1$, among the largest ever observed in glitches that lack a significant short-term exponential recovery. Remarkably, they have opposite signs: the second glitch has cancelled the effect of the previous increase in $\dot{v}$ and, actually, somewhat overshot it.

The jump in spin frequency after the first glitch appears have been recovered in $\sim 120 \mathrm{~d}$. The upper limit on $\ddot{v}$ after the first glitch implies that the jump in $\dot{v}$ could have been recovered, if at all, only on a much longer timescale and not until the second glitch occurred, $\sim 175 \mathrm{~d}$ after the first one. At the second glitch, an even larger spin-up occurred, accompanied by a significant flattening of the spindown trend. Thus, the spin up started with a sudden increase and then it slowly continued. A large and

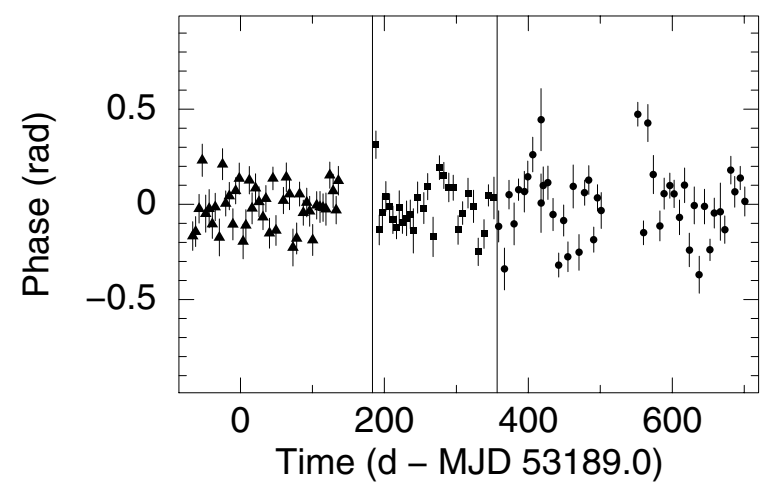

Fig. 2. Time residuals of Rossi-XTE RXSJ1708 observations from June 2004 to June 2006 after subtraction of pre- and post-glitch model N.1 and the polynomial post-glitch model N.2. Vertical lines indicate the assumed/inferred epochs of the glitches.

Table 1. Measured parameters for the two glitches detected in the Rossi$X T E$ data of RXSJ1708. $1 \sigma$ errors in the last digit are quoted in parenthesis.

\begin{tabular}{lcc}
\hline \hline Spin Parameter & post-glitch N.1 & post-glitch N.2 \\
\hline Epoch (MJD) & $53372(2)$ & $53546.0(8)$ \\
$v(\mathrm{~Hz})$ & $0.090887638(16)$ & $0.09088524(20)$ \\
$\dot{v}\left(\times 10^{-13} \mathrm{~s}^{-1}\right)$ & $-1.700(4)$ & $-1.536(7)$ \\
$\ddot{v} \times 10^{-22} \mathrm{~s}^{-3}$ & $<4.7$ & $-3.78(34)$ \\
MJD range & $53372-53545$ & $53546-53889$ \\
N. datapoints & 29 & 45 \\
rms (s) & 0.26 & 0.39 \\
$\Delta v / v\left(\times 10^{-6}\right)$ & $1.18(3)$ & $2.08(5)$ \\
$\Delta \dot{v} / \dot{v} \times 10^{-2}$ & $7.0(3)$ & $-10.35(34)$ \\
\hline
\end{tabular}

negative second derivative is found to be highly significant in the fits, which corresponds to a long-term recovery of $\Delta \dot{v}$ on a timescale $\simeq(503 \pm 48) \mathrm{d}$. We note that the $\ddot{v}$ term detected after the second glitch brings $\dot{v}$ back to the value it had before the first glitch in $\sim(159 \pm 26) \mathrm{d}$, and to steeper values afterwards. The extrapolated spin frequency at $500 \mathrm{~d}$ from the second glitch gives an additional spin-up $\sim(5.7 \pm 0.5) \times 10^{-7} \mathrm{~s}^{-1}$, while at the same epoch $\dot{v}$ returned to the value $\dot{v}(\Delta t=500 \mathrm{~d}) \simeq$ $-(1.70 \pm 0.02) \times 10^{-13} \mathrm{~s}^{-2}$ that it had after the first glitch.

\section{Discussion and conclusions}

RXSJ1708 experienced two new glitches, both with a large fractional amplitude, $\Delta v / v \sim 1.2 \times 10^{-6}$ and $2.1 \times 10^{-6}$ respectively, comparable to the so-called giant glitches observed from Vela and to the glitch previously detected from this source in May 2001 (Dall'Osso et al. 2003; Kaspi \& Gavriil 2003). This result strongly suggests that giant glitches seem to be the rule for this source.

It has been noticed (see Dall'Osso et al. 2003; Wang et al. 2000 , for details) that most glitch models are difficult to reconcile with observations of a growing number of glitches. In particular, all models requiring a catastrophic (i.e. widespread) unpinning of crustal superfluid (such as the vortex creep models, see Alpar et al. 1984, 1993, 1989) seem less promising, since they require simple correlations between the amplitudes and recovery timescales of glitches in a single source as well as an approximately constant $Q$-value (where $Q$ is the recovery fraction, see Dall'Osso et al. 2003), which is found not to be the case, in general, for radiopulsars. Although these problems can be partially 
solved by invoking local unpinning, this appears to be a rather ad hoc assumption (Jones 2002). Furthermore, it has been recently pointed out that the observation of long-period precession in a few pulsars is incompatible with pinning of crustal vortices (Link 2006).

Models where the internal angular momentum reservoir is in the NS core have some advantages with respect to the above points and can in principle explain a wider range of glitch properties (e.g. see Dall'Osso et al. 2003, for a more complete discussion). In this respect, it is worth noticing the unusually large jumps in the spindown rate of the two new events. A $~ 7 \%$ increase found after the first glitch would rule out the crustal superfluid as a momentum reservoir: the core superfluid - and a remarkable fraction of it - would necessarily be required to have been involved in the glitch. On the other hand, the large flattening that follows the second glitch is also very peculiar (although a residual steepening of the spindown trend, not recovered promptly, was also found in the May 2001 glitch from this same source, Dall'Osso et al. 2003). In general, it is not easy to explain long-term offsets from the secular spindown trend in glitch models based solely on crustal superfluid instabilities (Link et al. 1992), since these require permanent decoupling of some fraction of the internal superfluid from the bulk of the NS mass. More extreme difficulties are posed by the slowly recovered decrease in the spindown rate found in the second glitch: this apparently implies either a re-coupling of an internal component that was previously decoupled from the crust, or a corresponding decrease in the spindown torque.

A potential argument against core-based models arises when using $\dot{v}_{\mathrm{gl}}$ (the average spin-up rate caused by glitches) to estimate the fractional moment of inertia of the internal reservoir of angular momentum (Lyne et al. 2000; Dall'Osso et al. 2003). The parameters of the three large glitches detected so far from RXSJ1708 yield $\dot{v}_{\mathrm{gl}} \simeq 2.65 \times 10^{-15} \mathrm{~s}^{-2}$. Since the ratio $\dot{v}_{\mathrm{gl}} / \dot{v} \leq I_{\text {res }} / I_{\mathrm{c}}$, one derives $I_{\text {res }} / I_{\mathrm{c}} \geq 0.017$ where $I_{\text {res }}$ and $I_{\mathrm{c}}$ are the reservoir and stellar moments of inertia. This is comparable with the value inferred typically in glitching radio pulsars, and consistent with the crustal superfluid being the momentum reservoir (Lyne et al. 2000). However, we notice that the equality in the above formula obtains in the ideal case where the internal reservoir is completely decoupled from the crust. In the presence of coupling this component would also spin-down in between glitches at a rate $\dot{v}_{\text {res }}<\dot{v}$. Therefore, the following equality, $\dot{v}_{\mathrm{gl}} /\left(\dot{v}-\dot{v}_{\text {res }}\right)=I_{\text {res }} / I_{\mathrm{c}}$ should be considered instead. If glitches are produced once a critical rotational lag $v_{\mathrm{cr}}$ is reached, then $\dot{v}-\dot{v}_{\text {res }} \sim v_{\mathrm{cr}} / t_{\mathrm{g}}$, where $t_{\mathrm{g}}$ is the typical interglitch time. If we assume $I_{\text {res }} \simeq 0.1 I_{\mathrm{c}}$, as required to explain the changes in $\dot{v}$ found for the two new glitches, the measured $\dot{v}_{\mathrm{gl}}$ and a typical interglitch time $\leq 2 \mathrm{ys}$, we find $v_{\mathrm{cr}} \leq 1.6 \times 10^{-6} \mathrm{~s}^{-1}$. Whether such a low critical lag is meaningful on physical grounds is matter for future investigation (see Dall'Osso et al. 2003, for similar conclusions). An alternative explanation, in terms of starquakes and subsequent movements of cracked platelets, has been proposed for at least the large glitches of radio pulsars (Ruderman 1991; Ruderman et al. 1998). Quakes may be caused by the stress acting on the crust following the interaction in the core between superfluid neutrons and magnetic flux tubes threading the crust. Alternatively, the crustal strain that is accumulated during the growing of a twist in a magnetar's magnetosphere may represent a trigger in AXPs. Only the moving sector and its surroundings are affected, leaving the rest of the star mainly unperturbed (Jones 2002). The "local nature" of starquake models make them promising for reproducing the large variety of properties found in AXP glitches (Dall'Osso et al. 2003).

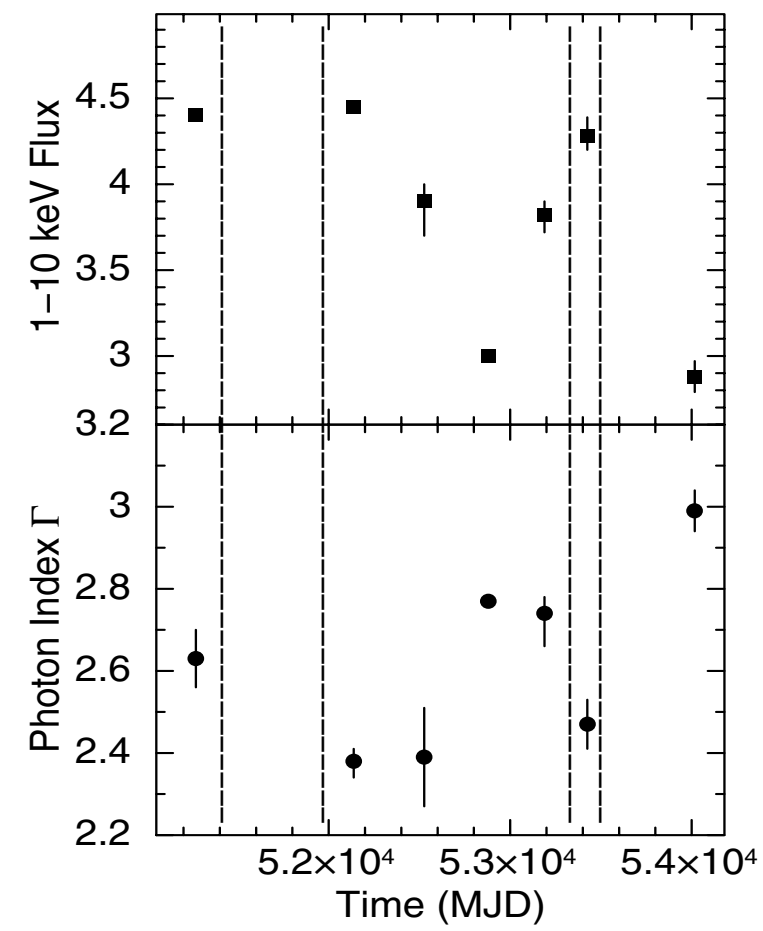

Fig. 3. Long-term spectral history of RXSJ1708 showing the correlated hardness-intensity variation. All reported fluxes are absorbed (with $N_{\mathrm{H}}=1.36 \times 10^{22} \mathrm{~cm}^{-2}$ for all the spectra) and in the $1-10 \mathrm{keV}$ energy range (see Götz et al. 2007, for further details). Vertical dashed lines mark the glitches detected in 1999, 2001, January and May 2005, from left to right respectively. Flux cross calibrations among instruments are less than $15 \%$ (note that the latest two measurements are both inferred from Swift XRT and imply a flux variability of more than 25\%).

In Rea et al. (2005a), we proposed that the observed correlation between the X-ray flux and the spectral hardness may be explained if the evolution is regulated by the change of a "twist" in the magnetosphere (see also Thompson et al. 2002). The evolving magnetic field is expected to fracture the crust at intervals, eventually causing an increased activity and large amplitude glitches. We found that observations collected until 2003 were consistent with a scenario in which the twist angle was steadily increasing before the glitch epochs, culminating with glitches and a period of increased timing noise, and then decreasing, leading to a smaller flux and a softer spectrum (see Fig. 3). Interestingly, the same model provides a natural explanation for the new period of glitching activity, that was foreseen in our previous papers. Nonetheless, we emphasise that while we do expect glitching activity corresponding of an increasing stress of the crust caused by a growing twist, glitches might also occur outside these epochs, in particular if, as stressed before, glitches with different properties (such as amplitude and recovery) may reflect a difference in triggering mechanism. We tried to estimate a reliable false alarm probability for the observed glitches to occur by chance close to the flux maxima displayed by observations, by assuming a uniform probability of flux values at glitch epochs. The false alarm probability depends on the maximum assumed flux range $\left(2.5 \times 10^{-11} \mathrm{erg} \mathrm{cm}^{-2} \mathrm{~s}^{-1}, 60 \%\right.$ more than that observed; see Fig. 3) and the given flux threshold value $\left(4.2 \times 10^{-11} \mathrm{erg} \mathrm{cm}^{-2} \mathrm{~s}^{-1}\right)$ above which glitches occur ${ }^{3}$. The above, somewhat conservative assumptions give a false alarm probability of $3 \%$. Based on the above considerations we believe

\footnotetext{
3 We implicitly assumed a uniform distribution for glitch epochs (which is likely not true).
} 
that the proposed link between timing and spectral properties of RXSJ1708 is rather good. However, given the poor knowledge of the true flux history and the not statistically independent nature of glitches, we believe that only future observations will be able to unambiguously confirm the link suggested by observations. Nonetheless, when the 1999 glitch is also added to the above considerations, the false alarm probability decreases to $1 \%$. Thus, if such correlated long-term variation will be further confirmed and discovered in other AXPs/SGRs, its X-ray monitoring might become an excellent tool to gather a deeper understanding of magnetars and, more generally, neutron star glitches.

Finally, we note that Dib et al. (2007) submitted a paper on an independent discovery of glitches from RXSJ1708 in the same dataset (after our original report at the Seattle Workshop on 27 th June $2007^{4}$ ): they considered our glitch N. 1 as a candidate rather than a true glitch. A detailed comparison between their results and ours is indeed premature and beyond the aims of the present work (Dall'Osso et al. 2007). Nonetheless, we note that a source of discrepancy is due to the use by Dib et al. (2007) of high-order frequency derivatives (used to identify glitches) in the presence of gaps in the phase-series. It is indeed not surprising that two out of the three candidate glitches reported by Dib et al. (2007) are found when data gaps are also present.

Acknowledgements. This work is partially supported at OAR through Agenzia Spaziale Italiana (ASI), Ministero dell'Istruzione, Università e Ricerca Scientifica e Tecnologica (MIUR - COFIN), and Istituto Nazionale di Astrofisica (INAF) grants. We acknowledge financial contribution from contract ASI-INAF I/023/05/0. S.Z. acknowledges support from a STFC (ex-PPARC) AF. D.G. acknowledges financial support from the French Space Agency (CNES).

\section{References}

Alpar, M. A., Langer, S. A., \& Sauls, J. A. 1984, ApJ, 282, 533

Alpar, M. A., Cheng, K. S., \& Pines, D. 1989, ApJ, 346, 823

Alpar, M. A., Chau, H. F., Cheng, K. S., \& Pines, D. 1993, ApJ, 409, 345

Beloborodov, A. M., \& Thompson, C. 2007, ApJ, 657, 967

Campana, S., Rea, N., Israel, G. L., Turolla, R., \& Zane, S. 2007, A\&A, 463, 1047

Dall'Osso, S., Israel, G. L., Stella, L., Possenti, A., \& Perozzi, E. 2003, ApJ, 599,485

Dall'Osso, S., et al. 2007, in prep.

Dib, R., et al. 2007, ApJ, in press [arXiv:0706 . 4156]

Götz, D., Rea, N., Israel, G. L., et al. 2007, A\&A, 475, 317

Israel, G. L., Covino, S., Stella, L., et al. 1999, ApJ, 518, L107

Israel, G. L., Oosterbroek, T., Stella, L., et al. 2001, ApJ, 560, L65

Israel, G. L., Covino, S., Perna, R., et al. 2003, ApJ, 589, L93

Jahoda, K., Swank, J. H., Giles, A. B., et al. 1996, Proc. SPIE, 2808, 59

Jones, P. B. 2002, MNRAS, 335, 733

Kaspi, V. M., \& Gavriil, F. P. 2003, ApJ, 596, L71

Kaspi, V. M., Lackey, J. R., \& Chakrabarty, D. 2000, ApJ, 537, L31

Link, B. 2006 [arXiv: astro-ph/0608319]

Link, B., Epstein, R. I., \& Baym, G. 1992, ApJ, 390, L21

Lyne, A. G., Shemar, S. L., \& Smith, F. G. 2000, MNRAS, 315, 534

Rea, N., Oosterbroek, T., Zane, S., et al. 2005a, MNRAS, 361, 710

Rea, N., Tiengo, A., Mereghetti, S., et al. 2005b, ApJ, 627, L133

Rea, N., Israel, G. L., Oosterbroek, T., et al. 2007, Ap\&SS, 308, 505

Ruderman, M. 1991, ApJ, 382, 587

Ruderman, M., Zhu, T., \& Chen, K. 1998, ApJ, 492, 267

Sugizaki, M., Nagase, F., Torii, K., et al. 1997, PASJ, 49, L25

Thompson, C., Lyutikov, M., \& Kulkarni, S. R. 2002, ApJ, 574, 332

Voges, W., Aschenbach, B., Boller, T., et al. 1996, IAU Circ., 6420, 2

Wang, N., Manchester, R. N., Pace, R. T., et al. 2000, MNRAS, 317, 843

Woods, P. M., \& Thompson, C. 2006, Compact Stellar X-ray sources, ed. W. Lewin, \& M. van der klis (Cambridge University Press)

Zane, S., et al. 2007, in The multicoloured landscape of compact objects and their explosive origin, Cefalu' (Sicily), 11-24 June 2006 (AIP), in press

\footnotetext{
${ }^{4}$ http://www. astroscu. unam.mx/neutrones/INT/ Workshop.html
} 